\title{
The effect of constant vs. varied encoding and massed vs. distributed presentations on recall of paired associates
}

\author{
MARIAN SCHWARTZ \\ University of Wisconsin-Madison, Madison, Wisconsin 53706
}

\begin{abstract}
The variables of coding (constant vs. varied) and spacing (massed vs, distributed) were factorially combined in an independent-groups design. Sixteen nominal pairs were presented twice, with the two presentations being either massed or distributed. A given nominal pair (e.g., AR-LE) was accompanied either by the same word-pair code on both presentations (e.g., arm-leg) or by a different code on each presentation (e.g., arm-leg; arrive-leave). On a subsequent recall test, the nominal stimulus only was shown. Better recall of the nominal response was found after constant coding and after distributed presentations, and there was no interaction between the spacing and coding variables. The results (a) support none of the current proposed explanations for the distributed practice effect, and (b) suggest that the critical factor in determining the probability of response recall is the strength of the strongest potential route to the response, not the number of different routes.
\end{abstract}

If a twice-presented pair is encoded differently on each presentation, is response recall more likely, or less likely, than if the pair had been encoded in the same way on both presentations? One view is that learning is facilitated by a stable, as opposed to a variable, encoding of the stimulus and of the association to the stimulus (e.g., Martin, 1972). Leading to the opposite prediction is the idea that repetition may be effective only to the extent that it enters new information into the memory store on successive presentations (Madigan, 1969); or the hypothesis that coding variation, by increasing the number of access routes to the response, will increase the probability of retrieval (Melton, 1967, 1970). Although the Melton and Madigan views have been associated primarily with free recall, the principle underlying them is testable in a variety of situations, including the paired-associate (PA) paradigm.

One purpose of the present experiment was to compare the effects of constant vs. varied encoding of pairs of verbal materials. Each nominal pair, which consisted of a bigram stimulus and a bigram response (e.g., AR-LE), was given two presentations. Along with the nominal pair, subject was supplied with what might be termed either a mediator or an elaborative encoding. This mediator, or code, consisted of a pair of associatively related words starting with the corresponding bigrams. For a constant-code group, a given nominal pair (e.g., AR-LE) was accompanied by the same word-pair code (e.g., arm-leg) on both presentations; for a varied-code group, a given nominal pair was accompanied by one code (e.g., arm-leg) on the first presentation and a different code (e.g., arrive-leave) on the second. In this way the varied-code group was, in effect, furnished two

This research was supported by a grant from the Graduate School of the University of Wisconsin. The author would like to thank Mary Jo Goecks for her help in collecting and analyzing the data. routes leading from the nominal stimulus to the nominal response, while the constant-code group was furnished the same route twice. The two groups were compared on a subsequent recall test in which the stimulus bigrams only were presented.

A second purpose of the present experiment was to investigate the distributed practice (DP) effect in the PA paradigm. The DP effect refers to the finding that repeated items are better recalled if the repetitions are spaced than if they are massed. In free recall, the effect has been found both with mixed-list and with unmixed-list designs (e.g., Underwood, 1970). In the PA paradigm, on the other hand, studies which have found the effect (Calfee, 1968; Greeno, 1964; Landauer, 1969; Peterson, Hillner, \& Saltzman, 1962; Peterson, Wampler, Kirkpatrick, \& Saltzman, 1963) appear to have used mixed lists exclusively. The present experiment used independent groups for the two spacing conditions. The finding of better recall with the distributed schedule would extend the generality of the DP effect and would be evidence against the "time-sharing" hypothesis as an explanation for its occurrence. That is, it has been suggested (Greeno, 1970; see also Melton, 1970) that, when the same item is presented twice in succession, the subject might use some of the time of the second presentation to process other items from the list. As such a strategy would favor distributed over massed items only if both types occurred in the same list, the time-sharing hypothesis could not account for a DP effect in the present experiment.

The occurrence of an interaction between the orthogonal variables of spacing and coding would be of particular relevance to the coding variation hypothesis espoused by Melton (1970) and Madigan (1969). Although it has more general implications, that hypothesis was formulated to account for the DP effect. 
While it does not specify the precise mechanisms by which distribution aids recall, the hypothesis includes the following points: (a) a distributed item occurs in more different contexts than does a massed item, (b) increasing the contextual variability for a given item permits more different cues to be stored with the item, or permits the item to be encoded along more different dimensions, or, in general terms, permits more new information to be entered into the memory store together with the item, thereby providing more access routes to the response, and (c) increasing the number of access routes to the response increases the probability of response retrieval. One implication of this hypothesis was indicated earlier; viz., if encoding is manipulated directly, rather than indirectly through distributed presentations, coding variation should facilitate recall. In terms of the present operations, this leads to the prediction of better recall in the varied-code than in the constant-code group. A second implication of the hypothesis is stated by Madigan $(1969$, p. 832$)$ as follows: "Given some experimental operation that will produce differences in coding of repetitions regardless of repetition lag, it should be possible to eliminate the effect of lag upon recall." In terms of the present operations, this implies that, if a DP effect is found, it should be limited to the constant-code condition.

In addition to investigating the effects of the spacing and coding variables upon recall of the nominal pairs, the present experiment investigated subsidiary questions concerning recall of the word-pair codes. In the varied-code condition, recall could be compared for the first-presented vs. the second-presented of the two codes supplied with each nominal pair. In both coding conditions, recall could be compared for word pairs differing in normative associative strength.

\section{METHOD}

\section{Materials}

Sixteen pairs of bigrams and 32 pairs of words were selected so that for each bigram pair, there were two corresponding word pairs. The stimulus and response members of each word pair started with the stimulus and response members, respectively, of the corresponding bigram pair. Selections were made from word association norms ${ }^{1}$ so that the stimulus term of each word pair was a normative stimulus, and the paired response term was one of the six most common normative responses to that stimulus. The 16 bigram pairs and their corresponding word pairs were as follows: AR-LE, arm-leg, arrive-leave; $\mathrm{BR}-\mathrm{SI}$, brother-sister, bronze-silver; $\mathrm{BU}-\mathrm{HO}$, bungalow-house, bugle-horn; $\mathrm{CA}-\mathrm{FL}$, carpet - floor, candle - flame; $\mathrm{CH}-\mathrm{RE}$, cherry-red, church-religion; $\mathrm{CO}-\mathrm{MI}$, cow-milk, coal-mine; DA-MO, dad-mom, day-month; GR-JU, grasshopper-jump, grapefruit-juice; HA-SA, happy-sad, hammer-saw; PA-WH, part-whole, pale-white; PO-RI, poor-rich, pony-ride; $\mathrm{SO}-\mathrm{BI}$, sour-bitter, song-bird; $\mathrm{ST}-\mathrm{RO}$, steal-rob, street-road; TE-BA, tennis-ball, tea-bag; TO-CI, town-city, tobacco-cigarette; WA-DR, water-drink, wash-dry.

Of the two word pairs starting with the same bigrams, the pair with higher normative associative strength (shown first in the above list) was assigned to Sublist $\mathrm{H}$, and the other pair was assigned to Sublist L. Normative associative strength was defined in terms of the percentage of all responses to its stimulus accounted for by the selected response; e.g., since leg accounted for $29 \%$ of the responses to arm in the word-association norms from which this pair was selected, and arrive accounted for $6 \%$ of the responses to leave, the former pair was assigned to Sublist $H$ and the latter to Sublist $L$. The mean percentage for the 16 word pairs in Sublist $\mathrm{H}$ was $33 \%$ (range $=9 \%-68 \%$ ); the mean for the 16 word pairs in Sublist L was $10 \%$ (range $=3 \%-24 \%$ ). In terms of frequency of occurrence in the language, the mean frequency in the Kucera and Francis (1967) corpus was, for the stimulus words, 101 in Sublist $\mathrm{H}$ and 100 in Sublist L; for the response words, 137 in Sublist $\mathbf{H}$ and 108 in Sublist L.

\section{Design}

The spacing and coding variables were factorially combined to form the four main groups: massed-constant, massed-varied, distributed-constant, and distributed-varied. In the massed condition, each of the 16 nominal (bigram) pairs was presented twice in succession. In the distributed condition, the list of 16 nominal pairs was presented once, and then repeated in the same order, so that there were always 15 other nominal pairs intervening between the two occurrences of a given nominal pair. A nominal pair was always accompanied by one of its two word-pair codes; either the same word pair was supplied on both presentations (constant-code condition) or a different word pair was supplied on each presentation (varied-code condition).

Each of the four main groups was divided into two subgroups. With in each constant-code group, subjects were assigned either to Subgroup HH (Sublist H word pairs on both presentations) or to Subgroup LL (Sublist L word pairs on both presentations). Within each varied-code group, subjects were assigned either to Subgroup HL (Sublist H word pairs on the first presentation, Sublist L on the second) or to Subgroup LH (vice versa).

\section{Procedure}

Subjects were run individually. The items were presented on a Stowe memory drum at 4-sec rate. Each bigram pair was typed in capital letters, with the first letter of the response bigram about $30 \mathrm{~mm}$ to the right of the first letter of the stimulus bigram. The accompanying word pair was typed in lower case below the bigram pair, with the first two letters of each word directly under the corresponding letters of the bigram.

Before the presentation trial, all subjects were given the same instructions, which included the following points: (a) on each presentation, subject should read aloud the stimulus bigram, stimulus word, response bigram, and response word in that order, and should try to remember what he had read; (b) the reason for presenting the word pairs was to help subject remember the bigram pairs; (c) on the later test, only the stimulus bigrams would be shown, and subject would be asked to recall, for each stimulus bigram, the correct response bigram and a corresponding word pair; (d) prior to the test, each of the 16 bigram pairs would be shown twice; the two presentations might or might not be consecutive, and the accompanying word pairs might or might not be the same. As part of the instructions, subjects were shown a sample bigram pair, SM-LA, along with two corresponding word pairs, small-large and smile-laugh.

After the 32 exposures which constituted the presentation trial, and before the recall test, a number-reading task was interpolated in order to eliminate any recency effect. The subject was instructed to read aloud, as fast as possible, from a sheet on which were 80 two-digit numbers which had been randomly selected without replacement from the set of all two-digit numbers and randomly ordered in four columns. After $30 \mathrm{sec}$ of number reading, subject was stopped and administered the written recall test. The test was divided into two parts which will be designated cued recall and supplementary recall, respectively. In cued recall, subject was given a recall sheet on which the 16 stimulus bigrams had been mimeographed, and was instructed to enter after each stimulus bigram the corresponding response bigram and word pair. In order to make the cued-recall task as comparable as possible for the varied-code and 
constant-code groups, the varied-code subjects were told to enter only one of the word pairs that had been shown with each bigram pair; specifically, the instruction was to enter whichever of the two word pairs subject remembered first, regardless of whether it had been the first or the second presented. All subjects were allowed 3 min for entry of the response bigrams and word pairs on the cued-recall test. Then subject was instructed to enter in the margin of the recall sheet, in any order, any additional words he could remember, and was allowed up to $3 \mathrm{~min}$ for this supplementary recall.

For generality, two orders of the 16 stimulus bigrams were prepared for the recall test, and two orders were prepared for the presentation trial. All four orders were random except that no sequence of two or more stimulus bigrams could be used in more than one order, and no stimulus bigram could be assigned to a given serial position in more than one order. There was, necessarily, a massed version and a distributed version of each presentation order. Within each of the eight subgroups, the two presentation orders and the two recall orders were used by equal numbers of subjects.

\section{Subjects}

The subjects were 96 students from an introductory psychology course, all of whom had English as their native language. Twelve subjects were assigned to each of the eight subgroups, using a block-randomization procedure. Data for an additional six subjects were discarded, five because of subject's failure to follow instructions, and one because of apparatus malfunction.

\section{RESULTS}

A preliminary question is whether the subjects in fact used the supplied word-pair codes in learning and recalling the nominal (bigram) pairs. Given that a response bigram was entered correctly (i.e., next to the appropriate stimulus bigram) during the cued-recall interval, the probability that a corresponding word pair would also be entered was $665 / 676$, or .984 . This strongly suggests that subjects were making use of the supplied codes rather than using idiosyncratic mediators or memorizing the nominal pairs by rote.

The main dependent variable was the number of correct bigrams entered during the cued-recall interval. As shown in Table 1, the mean was higher with distributed than with massed presentation, and higher with constant-code than with varied-code presentation. A 2 by 2 analysis of variance indicated that both main effects were significant, $F(1,92)=11.13, p<.01$ for the spacing variable and $F(1,92)=6.90, p<.05$ for the coding variable, while the interaction was not significant $(F<1)$. Separate 2 by 2 analyses were then performed

Table 1

Mean Number of Response Bigrams Correctly Entered During Cued Recall

\begin{tabular}{lccc}
\hline & \multicolumn{3}{c}{ Spacing Condition } \\
\cline { 2 - 4 } Coding & Both & Massed & Distributed \\
\hline Condition & 7.04 & 6.06 & 8.02 \\
Both & 7.81 & 6.54 & 9.08 \\
Vanstant & 6.27 & 5.58 & 6.96 \\
\hline
\end{tabular}

Table 2

Mean Number of Word Pairs Entered During Cued Recall by Subjects in the Varied-Code Condition

\begin{tabular}{lccc}
\hline & \multicolumn{3}{c}{ Subgroup } \\
\cline { 2 - 4 } Ordinal Position & Both & HL & LH \\
\hline Total & 6.13 & 7.09 & 5.16 \\
First Presented & 3.88 & 4.71 & 3.04 \\
Second Presented & 2.25 & 2.38 & 2.12 \\
\hline
\end{tabular}

on the constant-code and on the varied-code data, with spacing as one variable and subgroup as the other. In the constant-code condition, the effect of spacing was significant, $F(1,44)=9.48, p<.01 ;$ the effect of Subgroup HH (mean of 7.83 ) vs. Subgroup LL (mean of 7.79) was not significant $(F<1)$; the interaction was not significant $(F<1)$. In the varied-code condition, the effect of spacing failed to reach significance, $F(1,44)=2.74$, despite the absence of a Spacing by Coding interaction in the overall analysis; Subgroup HL (mean of 7.12) was significantly superior to Subgroup LH (mean of 5.42), $F(1,44)=4.23, p<.05$; and the interaction was not significant $(F<1)$.

In the varied-code condition, it may be assumed that whichever of the two corresponding word pairs subject entered during the cued-recall interval was in all likelihood the code he used to retrieve the response bigram. A breakdown of the correct word pairs is shown in Table 2. Following the procedure of Grant (1949), an analysis of variance was performed on the number of correct word pairs entered during cued recall by subjects in the varied-code condition. There were two between-subject variables: spacing (massed vs. distributed) and subgroup (HL vs. LH); and two within-subject variables: ordinal position (first-presented vs. second-presented of the two corresponding word pairs) and sublist membership (Sublist H vs. Sublist L). Consistent with the analysis of the response-bigram measure for the varied-code condition, spacing failed to reach conventional significance levels, $F(1,44)=3.09$, $.05<\mathrm{p}<.10$, while subgroup was significant, $F(1,44)=5.04, p<.05$. The two within-subject variables also were significant; i.e., of the two corresponding word pairs, subject was more likely to recall the first-presented than the second-presented, $F(1,44)=22.63, p<.001$, and more likely to recall the one from Sublist $H$ (mean of 3.42 ) than the one from Sublist $\mathrm{L}$ (mean of 2.71 ), $\mathrm{F}(1,44)=4.30, \mathrm{p}<.05$. The analysis allowed tests of three interactions-Spacing by Subgroup, Spacing by Ordinal Position, and Spacing by Sublist Membership-and $\mathbf{F}<1$ for all three.

In order to determine whether the effects of ordinal position and sublist membership were confined to entries made during the cued-recall interval, a count was made of the number of correct word pairs entered by varied-code subjects during the cued-recall and supplementary-recall intervals combined. This count, 
which included both of the corresponding word pairs if subject entered both, yielded relatively few additional items; the mean amounted to 7.38 , as compared with 6.13 for cued recall only. An analysis of variance performed on this measure did not change the dominance of the first-presented over the second-presented word pair, or the dominance of Sublist H over Sublist L.

\section{DISCUSSION}

Those subjects who were supplied with two encodings of each nominal pair showed poorer response recall than those supplied with the same encoding twice. Such a result is consistent with the idea that learning is facilitated by a stable rather than by a variable encoding of the stimulus and of the association to the stimulus (Martin, 1972) and is contrary to the view that multiple encodings should facilitate response retrieval (Madigan, 1969; Melton, 1970). Perhaps the simplest way to conceptualize the processes underlying this outcome is to assume that (a) in order for the nominal stimulus to cue the retrieval of the nominal response on the recall test, at least one retrieval route must have attained threshold strength, and that (b) the constant-code subjects had been furnished one relatively strong (because repeated) retrieval route for each nominal response while the varied-code subjects had been furnished two relatively weak (because not repeated) routes, the strengths of which were not additive.

Turning to the spacing variable, it should first be pointed out that the obtained superiority of distributed over massed presentation was not simply a reflection of a serial position effect. The occurrence of a primacy effect and/or a recency effect should favor the distributed schedule since, on the average, the first presentation of a nominal pair occurred earlier, and the second presentation occurred later, in the distributed than in the massed condition. Neither a primacy effect nor a recency effect was discernible, however, when correct response recalls were broken down by input serial position, with position being defined in terms of the order of presentation of the 16 nominal pairs. (The absence of a recency effect may be attributed to the interpolated number-reading task; the absence of a primacy effect may have stemmed from the paucity of time for rehearsal during the presentation trial, since almost all of the 4-sec interval was occupied by reading aloud.) It might also be noted that response recall at every one of the 16 input positions was higher in the distributed than in the massed condition. The superiority of the distributed schedule, therefore, may be attributed to spacing per se and not to a serial-position artifact.

The results extend the generality of the DP effect in the PA paradigm but support none of the various hypotheses that have been advanced to account for the superiority of distributed practice. First, the time-sharing mechanism (Greeno, 1970; Melton, 1970) could not account for the present DP effect, since independent groups were used for the two spacing conditions. Second, the coding variation hypothesis of Melton (1970) and Madigan (1969) has two implications for the present experiment. The results contradicted the implication that varied coding should facilitate recall, but are not clear-cut with respect to the implication that varied coding should eliminate the DP effect. On the one hand, the absence of a Spacing by Coding interaction $(F<1)$ in the overall analysis indicates that the DP effect was as strong in the varied-code as in the constant-code condition; on the other hand, the DP effect failed to reach significance in the separate analysis performed on the varied-code condition. On balance, the present findings must be considered unfavorable to the coding variation hypothesis. Finally, Bjork and Allen (1970) have divided theoretical explanations of the spacing or DP effect into two classes: Those that attribute the advantage of two spaced presentations over two massed presentations to better consolidation of the first presentation, and those that attribute the advantage to better encoding of the second presentation. In the present varied-code condition, the word pairs entered during the cued-recall interval indicate whether subject was retrieving the nominal pair from its first or from its second presentation. As reported earlier, subjects were significantly more likely to recall the first-presented word pair, but this was equally true in the massed and distributed conditions. The absence of an interaction between the variables of spacing and ordinal position $(F<1)$ suggests that the advantage of spacing falls equally on the first and second presentations. If this is so, then neither of the two classes of explanations described by Bjork and Allen (1970) would apply. An element of uncertainty, however, attaches to any inference drawn from the absence of an interaction with the spacing variable in the word-pair analysis since, as pointed out earlier, the main effect of spacing failed to reach conventional significance levels in that analysis $(.05<\mathrm{p}<.10)$.

Turning now to a consideration of the ordinal position effect per se, it is not apparent why subjects were more likely to recall the first-presented than the second-presented of two corresponding word pairs. It may be, however, that the mechanism underlying this effect is related to that underlying the priority effect described by Tulving and Watkins (1974). The priority effect refers to higher recall of a first list than of a second list, and apparently occurs (a) when the two lists represent an $A-B, A-C$ transfer paradigm but not when they represent an A-B, C-D paradigm, and (b) only when the first-list pairs are not tested prior to presentation of the second list. While the present materials do not conform to any standard transfer paradigm, the two sublists in the varied-code condition had an A-B, A-C 
component in each nominal stimulus and its two encodings, and the two sublists were presented without an intervening test.

With respect to the variable of sublist membership, it should first be noted that the requirements for selection of the word pairs did not allow the Sublist $H$ and Sublist $L$ pairs to be chosen from the extremes of normative associative strength. Rather, in terms of the percentage figures used to define degree of normative association, the word pair assigned to Sublist $\mathrm{H}$ was often little higher than the corresponding pair assigned to Sublist L. (For eight of the 16 corresponding word pairs, the difference was less than 15 percentage points.) Nevertheless, a significant difference was found in the varied-code condition, in favor of recall of the Sublist $\mathrm{H}$ word pairs. In the constant-code condition, neither mean number of response bigrams nor mean number of word pairs recalled showed any effect of sublist membership, but a pair-by-pair comparison revealed a consistent recall superiority for Subgroup HH over Subgroup LL in the six cases with the largest normative advantage (i.e., the six cases in which the associative strength of the Sublist $\mathrm{H}$ word pair exceeded that of the corresponding Sublist L word pair by at least 32 percentage points). Apparently, then, only sizable differences in normative associative strength would affect performance in the constant-code condition, while smaller differences were sufficient in the varied-code condition, perhaps because of its mixed-list presentation.

Finally, recall was found to be significantly higher in Subgroup HL than in Subgroup LH, both on the response-bigram measure and on the word-pair measure. In Subgroup HL, the variables of ordinal position and sublist membership were working in the same direction, giving Sublist $\mathrm{H}$ a double advantage over Sublist L; in Subgroup LH, these two variables were opposed, so that neither sublist had a double advantage over the other. In effect, therefore, Subgroup HL had been supplied with one relatively strong and one relatively weak retrieval route for each nominal pair, while Subgroup LH had been supplied with two retrieval routes which were more nearly equal in strength (see Table 2).

The superiority of constant over varied coding and the superiority of Subgroup HL over Subgroup LH both seem to point to the same conclusion. In the constant vs. varied comparison, a single code presented twice was more effective than two different codes presented once each; in the subgroup comparison, when two different codes were supplied for each nominal pair, a combination of one strong and one weak code was more effective than two codes of about medium strength. Both outcomes suggest that the critical factor in determining the probability of response recall is the strength of the strongest potential route to that response, not the number of different routes.

\section{REFERENCES}

Bjork, R. A., \& Allen, T. W. Spacing effect: Consolidation or differential encoding? Joumal of Verbal Learning and Verbal Behavior, 1970, 9, 567-572

Calfee, $R$. C. Interpresentation effects in paired-associate learning. Joumal of Verbal Learning and Verbal Behavior. $1968,7,1030-1036$.

Grant, D. A. The statistical analysis of a frequent experimental design. American Joumal of Psychology, 1949, 62, 119-122.

Greeno, J. G. Paired-associate learning with massed and distributed presentation of items. Journal of Experimental Psychology, 1964, 67, 286-295.

Greeno, J. G. Conservation of information-processing capacity in paired-associate memcrizing. Journal of Verbal hearning and Verbal Behavior, 1970, 9, 581-586.

Kucera, H., \& Francis, W. N. Computational analysis of present-day American English. Providence: Brown University Press, 1967.

Landauer, T. K. Reinforcement as consolidation. Psychological Review, 1969, 76, 82-96.

Madigan, S. W. Intraserial repetition and coding processes in free recall. Journal of Verbal Learning and Verbal Behavior, 1969 , $8,828-835$

Martin, E. Stimulus encoding in learning and transfer. In A. W. Melton \& E. Martin (Eds.). Coding processes in human memory. New York: Wiley, 1972.

Melton, A. W. Repetition and retrieval from memory. Science, $1967,158,532$.

Melton, A. W. The situation with respect to the spacing of repetitions and memory. Journal of Verbal Learning and Verbal Behavior, 1970, 9, 596-606.

Peterson, L. R., Hillner, K., \& Saltzman, D. Supplementary report: time between pairings and short-term memory. Joumal of Experimental Psychology, 1962, 64, 550-551.

Peterson, L. R., Wampler, R., Kirkpatrick, M. \& Saltzman, D. Effect of spacing presentations on retention of a paired associate over short intervals. Joumal of Experimental Psychology, 1963, 66, 206-209.

Tulving, E., \& Watkins, M. J. On negative transfer: Effects of testing one list on the recall of anothex. Joumal of Verbal Learning and Verbal Behavior, 1974, 13, 181-193.

Undervood, B. J. A breakdown of the total-time law in free-recall learning. Journal of Verbal Leaming and Verbal Behavior, 1970, 9, 573-580.

\section{NOTE}

1. One or more word pairs were selected from each of the following sources: (a) Bousfield, W. A., Cohen, B. H., Whitmarsh, G. A., \& Kincaid, W. D. The Connecticut free association norms. Studies on the mediation of verbal behavior. Tech. Rep. No. 35, University of Connecticut, November, 1961. (b) Deese, J. Free association norms for 599 words. Department of Psychology, Johns Hopkins University, no date, ditto. (c) Marshall, G. R., \& Cofer, C. N. Single-word free-association norms for 328 responses from the Connecticut cultural norms for verbal items in categories. In L. Postman and G. Keppel (Eds.), Norms of word association. New York: Academic Press 1970. (d) Riegel, K. F. Free associative responses to the 200 stimuli of the Michigan Restricted Association Norms. Analy sis of meaning with restricted association tests. Rep. No. 8, University of Michigan, April, 1965.

(Received for publication August 16, 1974: revision accepted October 5,1974 .) 